\title{
A new device for motion-aware ambulatory blood pressure measurement
}

\author{
L. T. D’Angelo, M. Lohmann, T. C. Lueth \\ Dept. Micro Technology and Medical Device Technology \\ Technische Universitaet Muenchen \\ Garching, Germany \\ Lorenzo.DAngelo@tum.de
}

\begin{abstract}
In this contribution, a system for motion-aware ambulatory blood pressure measurement is presented for the first time. Regular measurement of blood pressure is a key factor in controlling hypertension. However, blood pressure measurements in doctor's surgeries are random samples of a value varying during the day. $24 \mathrm{~h}$ ambulatory blood pressure measurement systems are used to get information about blood pressure changes in the course of a day. Measurement errors can occur due to movement artifacts, wrong posture during the measurement or absence of proper rest time prior to the measurement. The presented system monitors the user's motions and postpones planned measurements if proper rest is not detected. Additionally, it detects whether the user is in the proper upright position during measurement. Both, system concept and evaluations of BP measurement as well as movement and posture detection accuracy are presented.
\end{abstract}

Keywords - ABPM, NIBP, acceleration, motion, posture, artefact, ambulatory, blood pressure, portable

\section{INTRODUCTION}

Arterial hypertension, or high blood pressure (BP), is one of the greatest problems in industrialized nation's health systems. In Germany, $44 \%$ of women and $51 \%$ of men between 20 and 70 years are affected by it, i.e. have a systolic BP higher than $140 \mathrm{mmHg}\left(1 \mathrm{mmHg}=133.3 \mathrm{~N} / \mathrm{m}^{2}\right)$ and a diastolic BP higher than $90 \mathrm{mmHg}$ [1]. High BP is one of the main causes for cardiovascular diseases such as stroke, cardiac infarction, peripheral circulatory disorder, cardiac insufficiency, as well as renal insufficiency. It can thus lead to a lower life quality, to early invalidity or even to an early death. Apart from the personal consequences, high BP also leads to serious national economic problems. The mentioned diseases are the biggest causes of costs in the health system having a share of $15.6 \%$ [2]. The lost occupation time due to inability to work or early death in 2006 were accounted to $9.5 \%$ to cardiovascular diseases [1].

In Germany there is a huge gap between discovered (a hypertension which was diagnosed by a medic), treated (the patient gets a therapy against hypertension) and controlled (the therapy leads to a BP of $140 / 90 \mathrm{mmHg}$ or lower) hypertension. While the percentage of discovered hypertension amounts to $69 \%$ in the USA, it is $37 \%$ in Germany and thus among the last third in Europe. Also in respect to the treated and controlled quota (26\% and $7.8 \%$ ) Germany is not only clearly below USAmerican values (44\% and 54.5\%) but also in the lower group within Europe [3].
The reason for this can be found in the current diagnostic method (the measurement within the doctor's surgery), as it constitutes just a random sample of a value varying in the course of the day. Additionally it is prone to errors such as the white coat hypertension and can't detect masked hypertension. Among specific age groups, this can lead to wrong measurement in up to $60 \%$ of the cases [4].

To avoid these problems, a $24 \mathrm{~h}$ ambulatory BP measurement (ABPM) can be done. Measurement at home in regular intervals using home BP measurement devices is also possible. A prevention study carried out from 1984 to 1991 showed that the combination of regular BP measurements and non-medicamentous measures could raise the controlled hypertension quota by up to $35 \%$ [1]. With increasing number of ABPM done, algorithms for semiautomatic diagnosis generation and priorisation can be developed as it is already the case for electrocardiograms [5].

After the state of the art overview and task description, this paper will describe an ABPM system developed in order to measure BP in regular intervals taking into account the user's movements. Also, two experiments will be presented. The first experiment was done to assess the system's measurement accuracy. The second experiment was done to prove the system's ability to detect the user's movements and posture.

\section{A. State of the art in noninvasive BP measurement}

There are various noninvasive BP (NIBP) measurement systems for home or ambulatory use. In general, these can be classified as following:

- Cuff-based systems

$$
\begin{aligned}
& \circ \text { Oscillometric } \\
& \circ \text { Ascultatory (Korotkov method) }
\end{aligned}
$$

- Cuff-less systems

- Pulse Wave Velocity (PWV)

- Arteria Radialis Tonometric method

- Volume Clamp method

Most commercially available systems employ the oscillometric method, as well as systems in development [6]. The ascultatory method is the standard method in clinical practice and is more reliable than the oscillometric method. However, both methods cannot measure the BP continuously. 
Although developments aimed at textile measurements of movement or respiration rate $[7,8]$ are common, this is not true for BP, probably because of the size and energy consumption of available oscillometric sensor on the market.

The PWV method is based on a combined electrocardiogram (ECG) measurement and photoplethysmographic (PPG) pulse wave analysis $[9,10]$. The systolic BP is then calculated as being proportional to the pulse transit time [11], i.e. the time employed by the pulse wave to reach an extremity starting from the heart. The method requires a calibration using a cuff-based system. The Arteria Radialis Tonometric method calculates BP based on the pulse curve at the wrist and other parameters such as the wrist tissue elasticity and the radialis artery's size $[12,13]$.

Both cuff-less methods can measure BP continuously, but are still in research stadium. There is only one commercially available ABPM system employing the Arteria Radialis Tonometric method [14].

The volume clamp method employs a combination of cuff and PPG analysis. The cuff is inflated in order to keep a constant finger artery diameter and to reduce its transmural pressure to zero. The cuff pressure is then an indirect gauge of the BP. The method can measure continuously, requires calibration and shows good results [15] but hasn't pushed through yet.

In $24 \mathrm{~h} \mathrm{ABPM} \mathrm{practice,} \mathrm{the} \mathrm{system} \mathrm{is} \mathrm{set} \mathrm{up} \mathrm{by} \mathrm{the} \mathrm{clinical}$ personnel and then handed out to the patient. The system is composed of a measuring cuff and a small electronics box containing sensor evaluation and data storage components. Measurements are usually performed every 15 minutes during daytime and every 30 minutes at night. A market overview of available systems and their validation results is given by the European Society for Hypertension [12]. Table 1 presents some of them. Some devices come with software to set up and evaluate them more easily, e.g. MT200 by Schiller. Some offer printer terminals to export the data without the need of a personal computer (PC), e.g. the TM-2480 by A\&D.

Other research groups rather focus on a better integration of measurements in daily life than in the development of new measurement methods. The Fraunhofer IBMT Topcare healthcare kiosk offers a variety of vital parameter measurements outside home and thus an addition to home and doctor surgery measurement [17].

An assistance robot for processing and displaying vital parameters, in order to improve the compliance in taking measurements and the observation of alarming measurement results is suggested in [18]. The MINERVAA Project embedded vital parameter monitoring in airplane seats in order to improve the in-flight safety for people with health conditions.

Summing up, several products are available on the market for home and ABPM and new measurement methods are being developed. However, systems for home-use are to be actively used and are prone to measurement errors. This is especially true for wrist-cuff systems, where the quality of measurement results is strongly dependent on the current arm position and orientation. The device measures after a button is pushed without knowing whether the user had a rest time of $3-5 \mathrm{~min}$ and is in an upright position, both being important prerequisites for a correct measurement [19]. This problem is particularly pronounced in ABPM systems, which measure in rigid time intervals. In fact, for this reason ABPM results are not used singularly but calculating mean values over one hour and are yet far from being a standard component in hypertension therapy.

TABLE I. COMPARISON OF COMMERCIALLY AVAILABLE 24H ABPM SYSTEMS

\begin{tabular}{|c|l|l|l|l|}
\hline & \multicolumn{1}{|c|}{$[\mathbf{1}]$} & \multicolumn{1}{|c|}{$[\mathbf{2 ]}$} & \multicolumn{1}{|c|}{$[\mathbf{9 ]}$} & \multicolumn{1}{|c|}{} \\
\hline Measurement method & OSC & OSC & OSC & ASC \\
\hline Memory slots & 240 & 600 & 300 & $\mathrm{nk}$ \\
\hline $\begin{array}{l}\text { Max measurements } \\
\text { without recharging }\end{array}$ & $\mathrm{nk}$ & 300 & $\mathrm{nk}$ & $\mathrm{nk}$ \\
\hline Battery type & $3 \times \mathrm{xA}$ & $4 \mathrm{xAA}$ & $3 \mathrm{xAAA}$ & $2 \mathrm{xAA}$ \\
\hline Mass without cuff (g) & 255 & 350 & 215 & 198 \\
\hline Dimensions (mm) & $\begin{array}{l}250 \times 100 \\
\mathrm{x} 70\end{array}$ & $\begin{array}{l}124 \mathrm{x} 82 \mathrm{x} \\
33.5\end{array}$ & $\begin{array}{l}100 \mathrm{x} 72 \mathrm{x} \\
27\end{array}$ & $\begin{array}{l}101 \times 69 \mathrm{x} \\
28\end{array}$ \\
\hline Data interface & $\mathrm{nk}$ & $\begin{array}{l}\mathrm{RS} 232, \\
\text { optical } \\
\text { cable }\end{array}$ & $\mathrm{RS} 232$ & USB \\
\hline Special features & & & & $\begin{array}{l}\text { Speech } \\
\text { recording }\end{array}$ \\
\hline BHS certification & $\mathrm{A} / \mathrm{A}$ & $\mathrm{B} / \mathrm{B}$ & $\mathrm{B} / \mathrm{A}$ & $\mathrm{B} / \mathrm{B}$ \\
\hline
\end{tabular}

$\mathrm{OSC}=$ Oscillometric $; \mathrm{ASC}=$ Ascultatory; $\mathrm{nk}=$ not known; $\mathrm{AA}=\mathrm{AA}$ sized battery $; \mathrm{AAA}=\mathrm{AAA}$ sized battery

\section{B. Task description}

Pervasive medical devices can contribute to a measurement with information about the user activity and movement and can integrate with available handhelds for user interface [20,21]. The goal of the presented project is to develop a system able to acquire BP values similarly to ABPM systems using the oscillometric method. In addition to this, it should monitor the wearer's movements and adapt the measurement intervals within a given limit in order to preferably take measurements after the user had a sufficient rest period. It should also monitor the movements during the measurement in order to avoid movement artifacts. It should be intuitive to use and able to store the measurements with a timestamp as well as exporting them via a radio link.

\section{SYSTEM CONCEPT}

\section{A. Static system concept description}

The system is composed of a BP cuff, an electronics box and a radio data receiver. The BP cuff can be a standard BP cuff and the receiver unit can be similar to the one described in [22]. The electronics box can also exchange data and be reprogrammed using a PC. A system composition overview can be seen in Fig. 1. In the following, the electronics box will be described. The box contains a control module, a standard OEM BP measurement module and a battery. The user interface on the box gives the ability to manually start a measurement and to offer a direct qualitative feedback as well as information about battery and memory status. The control module inside the box (Fig. 2) contains a microcontroller, an accelerometer, a radio chip, a real-time clock, a relay as well as an external interface (serial connection) and energy management. 


\section{B. Dynamic system concept description}

The system can be used for 24h ABPM measurement and for personal use. In the first case, the system is set up and the data is read out by a clinician, in the second case those tasks are fulfilled by the user.

In this section, the processes running on the system's control module are described. A main processes overview is given in Fig. 3.

After switching it on, it is possible to configure the system over the serial interface for $10 \mathrm{~s}$. This includes setting the current time for the real time clock, setting the next measurement time as well as the desired measurement interval and calibrating the accelerometer. During calibration the user has to carry the box in the preferred way and remain in rest. Thus, the box orientation on the body and a quiescent value are determined. The calibration has to be done once, unless the user wants to change the orientation in which he carries the box on his body.

After the system setup phase or 10s after the start, the main process waits for either a manually started measurement or an automatic measurement. In case of a manually started measurement, the BP measurement module is activated, a measurement is performed and values obtained are stored together with the current posture value. The posture value is a binary value which states whether the patient was in an upright position or not. Non upright is defined as a deviation of the absolute acceleration quiescent value of more than $0.6 \mathrm{~g}_{\mathrm{n}}\left(1 \mathrm{~g}_{\mathrm{n}}=\right.$ $9.81 \mathrm{~m} / \mathrm{s}^{2}$ ), which corresponds to a body inclination of $24^{\circ}$ or less in respect to the floor. In case of an automatic measurement, the user's movement status is determined averaging the highest accelerations occurred within one second over ten seconds.

If this value does not exceed the quiescent value by more than $0.1 \mathrm{~g}_{\mathrm{n}}$ for more than $3 \mathrm{~min}$, the measurement is started and stored together with the posture value. If the value is too high, the measurement is postponed until the measurement value is low enough. If the value does not get low enough for a period of time $t_{\text {shift }}$, the measurement is carried out nonetheless and the value is stored together with the posture value and a value indicating the user has been moving.

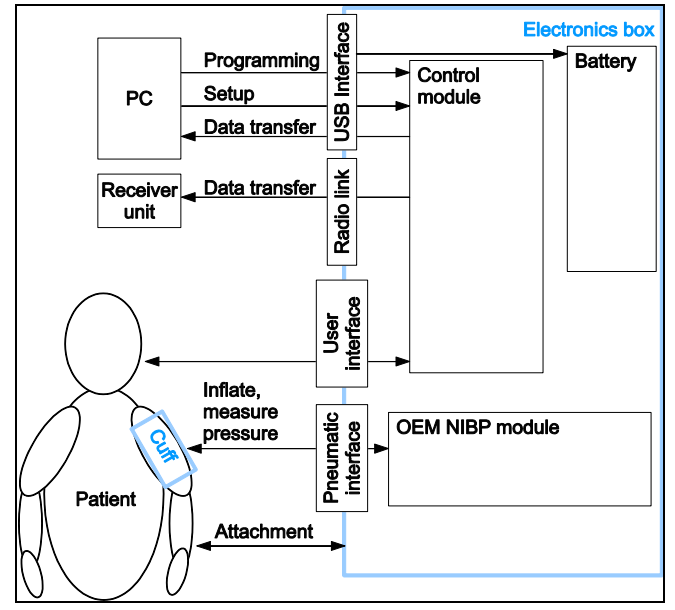

Figure 1. System compsition overview.

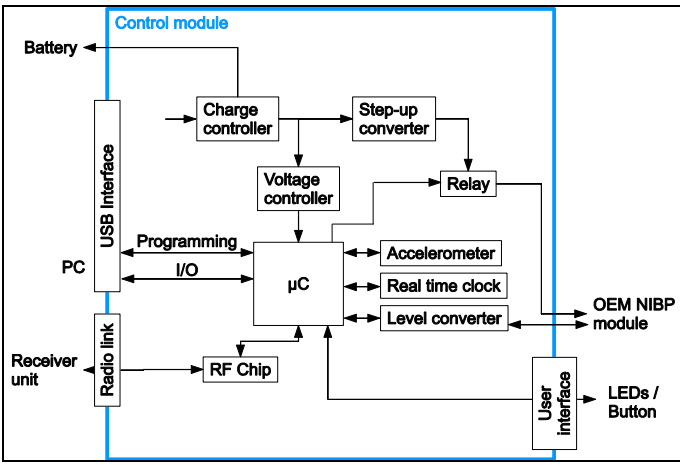

Figure 2. Description of the system's parts and interfaces.

The value $t_{\text {shift }}$ is calculated as follows, given the measurement interval $t_{\text {int }}[\mathrm{min}]$ :

$$
t_{\text {shift }}=\frac{t_{\mathrm{int}}}{3}+3[\mathrm{~min}]
$$

After each measurement the device also gives an immediate qualitative feedback to the user about the measured value based on the WHO recommendation on BP levels (units are $\mathrm{mmHg}$ ):

- normal (systolic $\mathrm{BP}<130$ \& diastolic $\mathrm{BP}<85$ )

- high $(130<=$ systolic $\mathrm{BP}<139 \& 85<=$ diastolic $\mathrm{BP}<89)$

- hypertension (systolic BP>=140 \& diastolic BP>=90).

Between measurements, the device searches for nearby receivers and, if one is found, the data is transferred to it and erased from the internal memory. After 30 s of inactivity the device switches to a sleep mode.

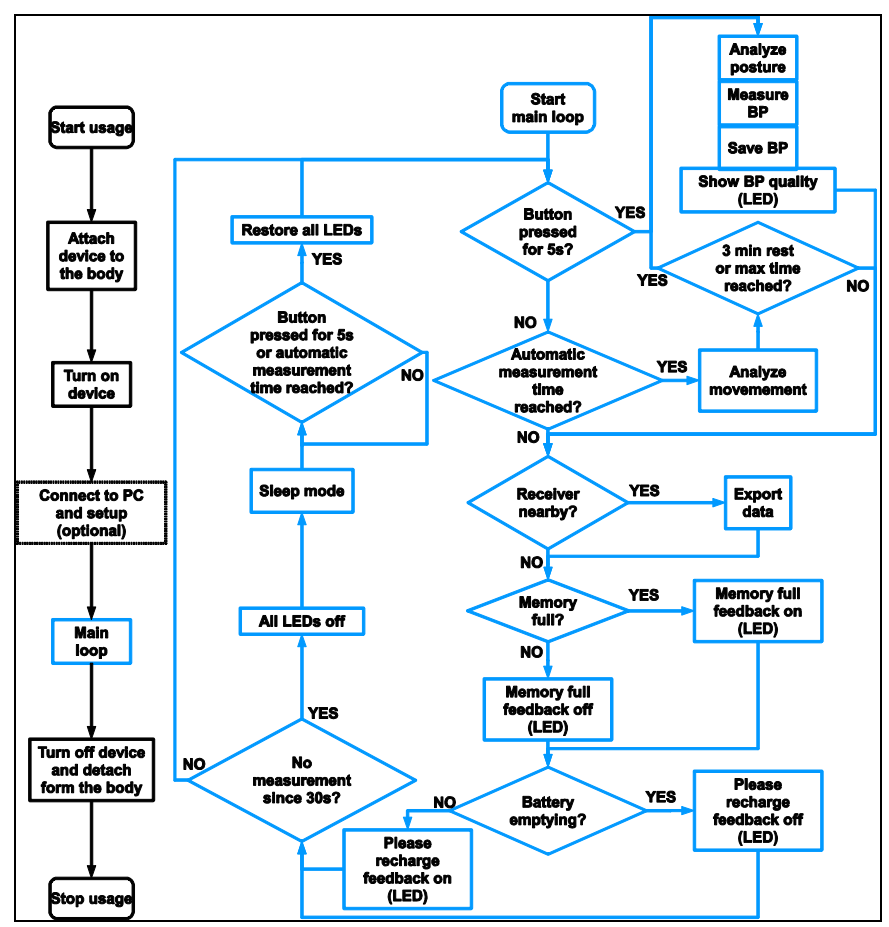

Figure 3. Description of the system's processes. 


\section{SySTEM EVALUATION}

For evaluation, the presented system was set up as described in section III.A and two experiments were conducted. The first one, described in section III.B, had the purpose of evaluating the reliability of the measurements carried out by the system. The second one, described in section III.C, was conducted to evaluate the sensor's ability to detect user movement and posture and thus to find the best point in time for a measurement.

\section{A. Materials and methods}

The prototype built to evaluate the presented system concept is shown in Fig. 4. The electronics box is built from modified standard components; the user feedback is given by LEDs. Manual measurement can be started pushing a button for $5 \mathrm{~s}$. The device is turned off and on by a switch on the side. As described before, this switch is not needed during the day as the device gets into sleep mode autonomously and wakes up upon reaching a measurement time or pushing the button.

The device's dimensions $(126 \times 86 \times 32 \mathrm{~mm})$ and mass $(350 \mathrm{~g})$ are comparable to the smallest ABPM devices on the market (see also table 1). The device is fixed to the body using a necklace and abdominal belt. The necklace is also used to fix the tube connected to the measurement cuff. As a battery, a $1600 \mathrm{mAh}$ capacity lithium polymer battery supplying a voltage of $3.7 \mathrm{~V}$ was chosen. The used BP module is a NibScan OEM Module by Medlab GmbH. It contains microcontroller pump, valve, pressure sensor, cuff tube attachment and a pin connector implementing a digital interface for measurement triggering and data output. The cuff is also a standard BP measurement cuff by Medlab $\mathrm{GmbH}$, certified for arm circumferences between 27 and $35 \mathrm{~cm}$.

The controller module's core is an AtMega644V 8bit microcontroller by Atmel and a $2.4 \mathrm{GHz}$ nanoLOC TRX radio transceiver by Nanotron Technologies. The microcontroller comes with $2 \mathrm{kB}$ EEPROM, which is sufficient for the storage of 180 data packages. The installed SMB380 accelerometer by Bosch measures accelerations in all three spatial axis with a 10 bit resolution and a range set to $\pm 8 \mathrm{~g}$. As real time clock, a RV-8564-C2 module by MicroCrystal Switzerland is used. The controller module also contains a XC6204 voltage regulator by Torex converting the batteries $3.7 \mathrm{~V}$ into the $2.5 \mathrm{~V}$ required by the microcontroller and a MAX1797 step up converter by Maxim generating $5 \mathrm{~V}$ required by the $\mathrm{BP}$ measurement module. A PCA9306 level converter by Texas Instruments is used to convert serial TTL levels between microcontroller and BP measurement module. The microcontroller can interrupt the BP measurement module's current supply switching a G6K-2F$3 \mathrm{VCD}$ relay by Omron.

\section{B. Evaluation of measurement accuracy}

\section{1) Motivation}

In this experiment, the measurement accuracy of the developed system was to be evaluated following the international AAMI (Association for the Advancement of Medical Instrumentation) standard, which represents the gold standard proof of accuracy for BP measurement systems together with the BHS (British Hypertension Society) standard [16].

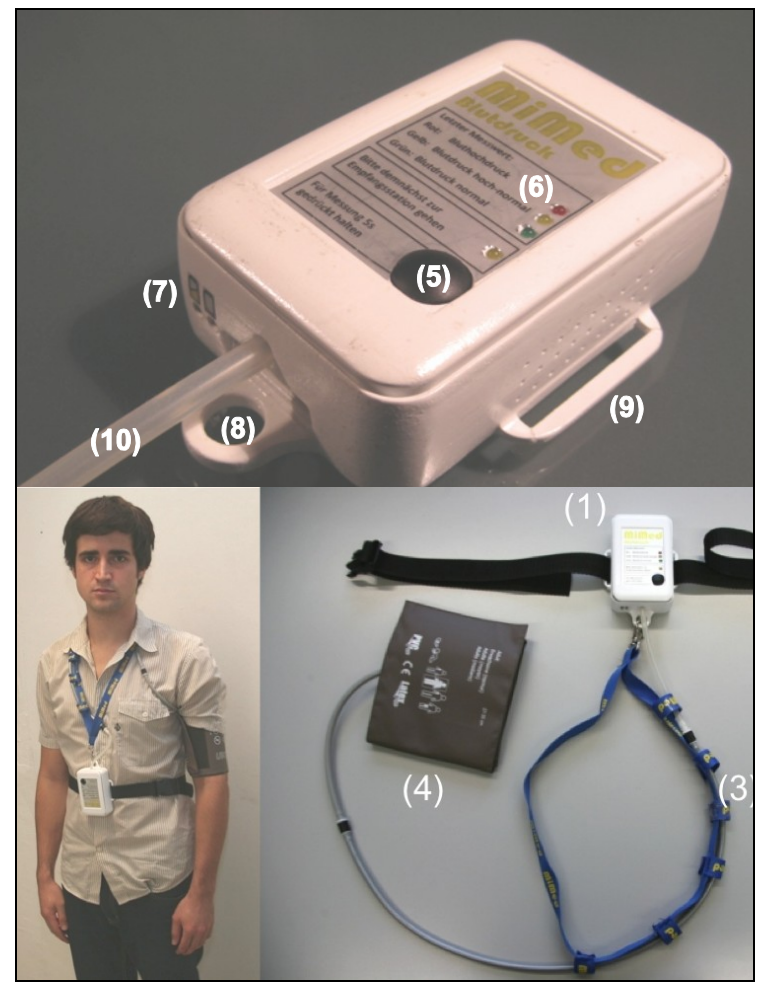

Figure 4. Top: Detailed view of the electronic box. Bottom left: usage example. Bottom right: overview of the device for motion-aware ABPM. (1) Electronics box, (2) abdominal belt, (3) necklace, (4) BP measurement cuff,

(5) manual measurement start button, (6) WHO qualitative feedback LEDs,

(7) battery status LEDs, (8) necklace attachment, (9) abdominal belt attachment, (10) cuff inflation tube.

\section{2) Setup}

The AAMI standard requires the following experimental setup:

- $\quad$ Mercury manometer calibrated to $\pm 1 \mathrm{mmHg}$ accuracy as reference device

- At least 85 test subjects, 3 measurements per subject and device

\section{- Heterogeneous test group}

For practical reasons, the used test setup deviated from the standard as follows (and is thus not valid for certification): as test subjects, 21 male subjects aged 24-34 were chosen, and 2 measurements were done per subject and device. As reference device, an ES-P2000 by Terumo was chosen instead of a mercury manometer. The ES-P2000 is certified according to the BHS standard.

Every measurement was taken first with the new device and then with the ES-P2000 in an upright sitting position and after at least 3 minutes of rest. The cuff was placed $2-3 \mathrm{~cm}$ above the arm pit and the arm was placed on a table slightly inflected. The measurements with the new device were started manually and read out on a PC using the external interface. The measurements with the reference device were read out on the device's display. The values for one subject were discarded 
because the cuff size did not fit the arm size, leaving 40 value pairs for evaluation.

The evaluation criteria were identical to the AAMI standard criteria:

1. The new device's values must be within $\pm 10 \mathrm{mmHg}$ of the reference device's values in at least $95 \%$ of $\mathrm{N}$ measurements and within $\pm 5 \mathrm{mmHg}$ of the reference device's values in at least $85 \%$ of all measurements, i.e.:

$0,95 \leq \frac{1}{N} \sum_{i=1}^{N} x_{i} ; x_{i}=\left\{\begin{array}{l}1||\left(B P_{\text {test }, i}-B P_{\text {ref }, i}\right) \mid \leq 10 \mathrm{mmHg} \\ 0||\left(B P_{\text {test }, i}-B P_{\text {ref }, i}\right) \mid>10 \mathrm{mmHg}\end{array}\right.$

$0,85 \leq \frac{1}{N} \sum_{i=1}^{N} x_{i} ; x_{i}=\left\{\begin{array}{l}1||\left(B P_{\text {test }, i}-B P_{r e f, i}\right) \mid \leq 5 m m H g \\ 0||\left(B P_{\text {test }, i}-B P_{\text {ref }, i}\right) \mid>5 m m H g\end{array}\right.$,

for $\mathrm{BP}_{\text {test, } \mathrm{i}}$ being the $\mathrm{BP}$ value (systolic or diastolic) taken in the $\mathrm{i}^{\text {th }}$ measurement with the new device and $\mathrm{BP}_{\text {ref,i }}$ being the $\mathrm{BP}$ value taken in the $\mathrm{i}^{\text {th }}$ measurement with the reference device.

2. $\mu_{\mathrm{BP}}$ (average systolic or diastolic measured BP difference) must be smaller than $\pm 5 \mathrm{mmHg} ; \quad \sigma_{\mathrm{BP}}$ (standard deviation of systolic or diastolic measured BP difference) must be smaller than $8 \mathrm{mmHg}$, i.e.:

$\left|\mu_{B P}\right|=\left|\sum_{i=1}^{N} \frac{d_{B P, i}}{N}\right|<5 m m H g$

$\sigma_{B P}=\sqrt{\frac{1}{N-1} \sum_{i=1}^{N}\left(d_{B P, i}-\mu_{B P}\right)^{2}}<8 m m H g$,

for $\mathrm{d}_{\mathrm{BP}, \mathrm{i}}$ being the systolic or diastolic BP difference taken in the $\mathrm{i}^{\text {th }}$ measurement.

3) Results

In the following, the results are given for both AAMI standard criteria:

1. Criterion

- Systolic values were within $\pm 10 \mathrm{mmHg}$ in $97,5 \%$ of the cases

- Systolic values were within $\pm 5 \mathrm{mmHg}$ in $85 \%$ of the cases

- Diastolic values were within $\pm 10 \mathrm{mmHg}$ in $82,5 \%$ of the cases

- Diastolic values were within $\pm 5 \mathrm{mmHg}$ in $65 \%$ of the cases

2. Criterion

- $\mu_{\mathrm{BP}, \text { systolic }}=-0,375 \mathrm{mmHg}, \sigma_{\mathrm{BP}, \text { systolic }}=5,87 \mathrm{mmHg}$
- $\mu_{\mathrm{BP} \text {,diastolic }}=-0,8 \mathrm{mmHg}, \sigma_{\mathrm{BP} \text {, diastolic }}=8,52 \mathrm{mmHg}$

Thus, AAMI standard criteria are met for systolic, but not for diastolic measurement. The Bland-Altman plots for both measurements are shown in Fig. 5 and Fig. 6.

The insufficient performance regarding the diastolic values despite the use of an OEM module has presumably two reasons (systematic measurement errors):

- As can be seen, in Fig. 7, plotting the measurement deviations over the test subject's arm circumference, a high number of deviations occurred with test subjects with an arm circumference of $27 \mathrm{~cm}$. This was the lowest allowed value for the cuff employed in the experiment. Due to errors in measuring the circumferences, some may actually have been lower.

- The reference device's accuracy may be too low, as the BHS certification criteria are not as strict as the AAMI criteria.

These can be solved using measurements done by a medical doctor and a calibrated mercury manometer as reference in future experiments.

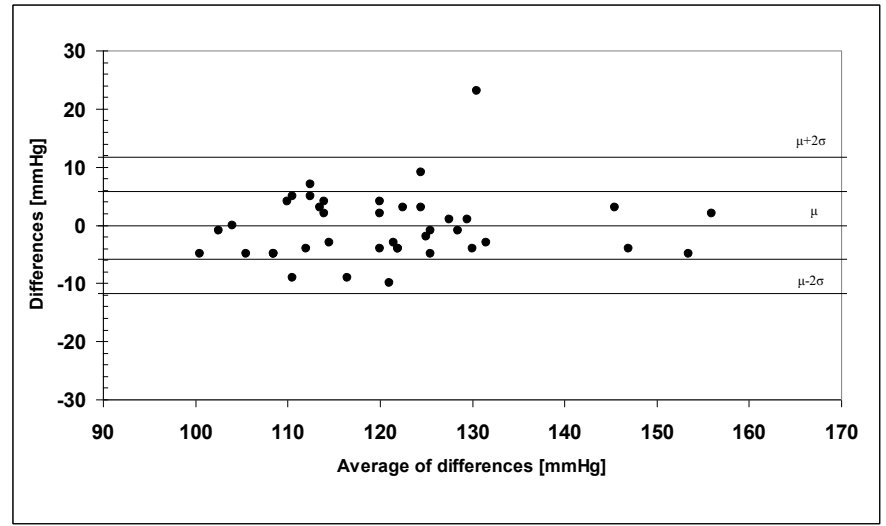

Figure 5. Bland-Altman plot showing the differences between the presented device and the refrence device measuring systolic BP over their averages .

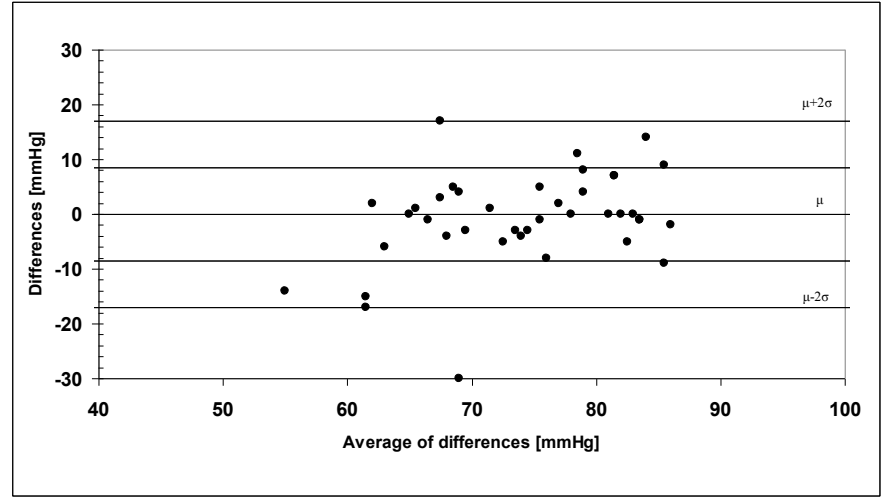

Figure 6. Bland-Altman plot showing the differences between the presented device and the refrence device measuring diastolic BP over their averages . 


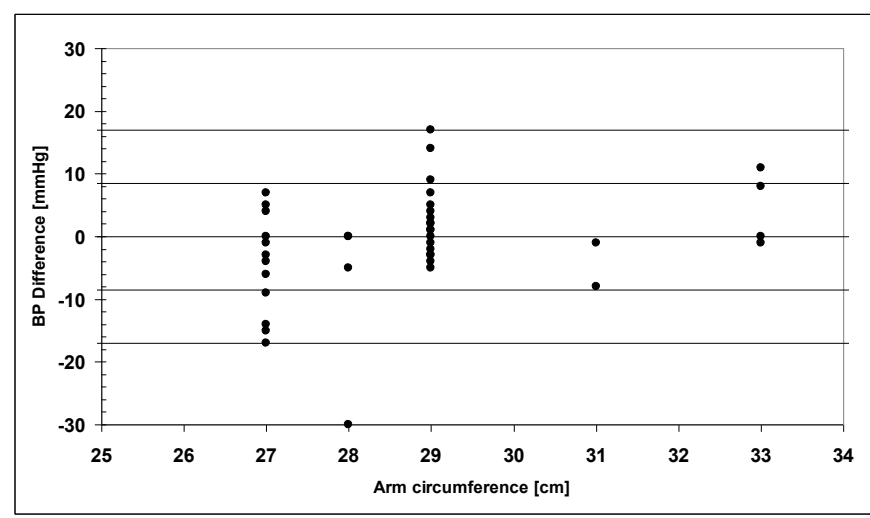

Figure 7. Differences between the presented device and reference device measuring diastolic BP over test subject's arm circumferences.

\section{Evaluation of movement and posture detection}

\section{1) Motivation}

This experiment was conducted to prove that the new device is able to detect movement (3 minutes of rest required for measurement) state and posture (upright position required for measurement) of the user and is able to postpone measurement when needed. The goal was to reach a concordance in movement status and posture detection of at least $90 \%$ and at least $80 \%$ of positive answers to the subjective user evaluation questionnaires.

\section{2) Setup}

As there is no standard available for this proof, the following setup has been designed: the measurement device is set to a measurement interval of 15 minutes, attached to a test subject and a first measurement is started in upright sitting position. After this, the test subject is requested to follow an arbitrary activity (chosen by the subject, e.g. playing Wii) for 50 minutes. During or shortly after every measurement taken by the device the subject is asked to write down a note about his movement status before and his posture during the measurement. After the 50 minutes, the test subject gives back the measurement device and the notes taken. Then the subject answers a questionnaire asking

- The device constrains me in my activity.

- The device adapts the measurement times well to my activity.

Both questions could be answered with "agree" or "not agree". The experiment was conducted with 3 male and 2 female subjects aged 26-28 resulting in 5 rest and 15 ambulatory measurements (recorded but not further evaluated) and 5 answered questionnaires.

\section{3) Results}

In $93 \%$ of all cases the device detected correctly that the subject was moving before a measurement. In $100 \%$ of all cases the device detected the subject's posture (lying/standing) correctly during the measurement. To the question "The device constrains me in my activity", $100 \%$ of the test subjects answered "not agree". To the question "The device adapts the measurement times well to my activity" $80 \%$ of the test subjects answered "agree". A plot of the results is given in Fig. 8.

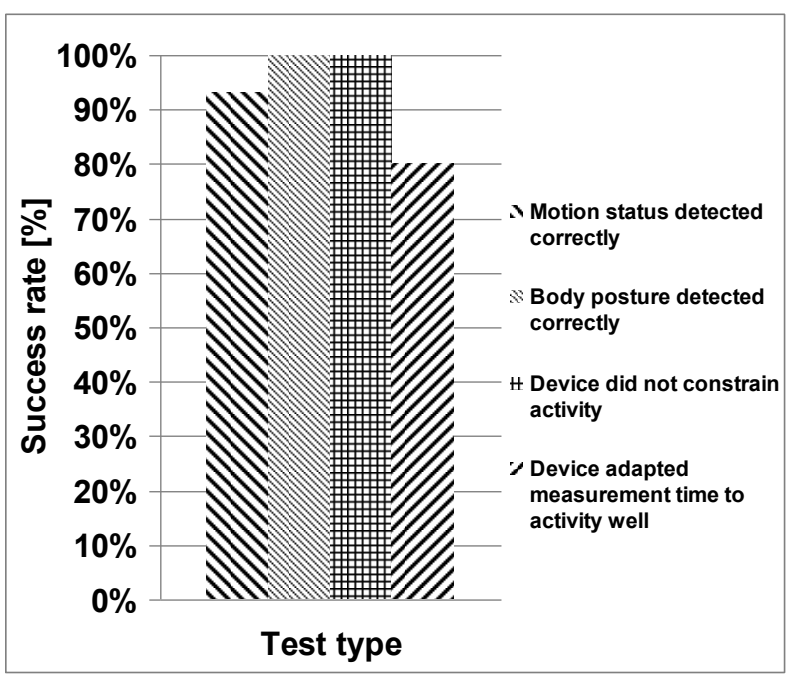

Figure 8. Results of the movement and posture detection assessments.

The postponement of a measurement in case of movement was verified by all test subjects, i.e. every measurement that was tagged with a movement warning was postponed by 8 minutes and every measurement without had been carried out in a time span before.

Thus, the experiment proved that the requirements are fully met.

\section{CONCLUSIONS}

A new device for motion-aware ABPM is presented for the first time. The system has the advantage of being able to detect the user's movement state and posture and thus to adapt the time of measurements to his activity. This feature avoids taking measurements without a proper previous rest period or in nonupright position. If the desired measurement interval does not allow further postponement, a measurement is taken nonetheless, but information about movement or wrong posture is added to it. The device can store up to 180 measurements and can be read out via a radio link or a direct link with a PC. In two experiments, the measurement accuracy as well as the movement and posture detection have been evaluated. In future steps, the measurement accuracy evaluation should be repeated with more test subjects and a calibrated mercury manometer as reference device. Also, extensive ergonomic tests with more test subjects and extensive questionnaires should be carried out. It should also be determined whether the use of this device in ABPM can allow using bigger measurement time intervals. The developments in continuous cuff-less NIBP monitoring allow the hope for a future development of a certified, motion-aware cuff-less ABPM device.

\section{ACKNOWLEDGMENT}

Within the research consortium of the Bavarian Research Foundation (BFS) „FitForAge“ a team of scientists and engineers affiliated to 13 departments of the Bavarian universities Erlangen-Nürnberg, München, Regensburg and Würzburg works together with 25 industrial partners on the development of products and services for the aging society. 
The scope of the research consortium is to develop technology based solutions which will help elderly people in their future living environment comprising home and workplace as well as in communication and transportation. Eventually not only elderly people but also all social groups should profit from these solutions.

\section{REFERENCES}

[1] Statistisches Bundesamt, Robert Koch Institut, „Hypertonie“ [Hypertension] in Gesundheitsberichterstattung des Bundes, 2008, Heft 43, Berlin

[2] Statistisches Bundesamt, "Gesundheit - Ausgaben, Krankheitskosten und Personal 2004" [Health - Costs, Health Expenditure and Personnell 2004], 2006, Wiesbaden

[3] Wolf-Maier K, Cooper RS, Kramer H et. al., "Hypertension Treatment and Control in Five European Countries, Canada, and the United States“" in Hypertension 2004;43;S.10-17

[4] Hozawa A, Ohkubo T., Kikiya Mho et. al., „Blood Pressure Control Assessed by Home, Ambulatory and Conventional Blood Pressure Measurements in the Japanese General Population: the Ohasama Study" in Hypertension Res., 2002 Jan, 25(1):S.57-63.

[5] D'Angelo L.T., Tarita E., Zywietz T.K., Lüth T.C. (2010) : A System for Intelligent Home Care ECG Upload and Priorisation, in 32nd Annual International Conference of the IEEE Engineering in Medicine and Biology Society, Medline, U.S. National Library of Medicine, pp. 21882191.

[6] Dai, H.; D'Angelo, L.T.; Lueth, T.: "Continuous blood pressure monitor with wireless interface," Robotics and Biomimetics (ROBIO), 2010 IEEE International Conference on, vol., no., pp.36-40, 14-18 Dec. 2010.

[7] D'Angelo L.T., Weber S., Honda Y., Thiel T., Narbonneau F., Lüth T.C. (2008) : A system for respiratory motion detection using optical fibers embedded into textiles, in 30th Annual International Conference of the IEEE Engineering in Medicine and Biology Society, Medline, U.S. National Library of Medicine, pp. 3694-2697.

[8] Niazmand K., Jehle C., D'Angelo L.T., Lüth T.C. (2010) : A New Washable Low-Cost Garment for Everyday Fall Detection, in 32nd Annual International Conference of the IEEE Engineering in Medicine and Biology Society, Medline, U.S. National Library of Medicine, pp. 6377-6380.

[9] Guo DG, Tay FEH, XU L et al.,"A Long-term Wearable Vital Signs Monitoring System using BSN"in Digital System Design Architectures, Methods and Tools, 2008. DSD '08. 11th EUROMICRO Conference on, 2008 Sept 3-5: 825 - 830

[10] Espina J, Falck T, Muehlsteff J et al., "Wearable Body Sensor Network towards Continuous Cuff-less Blood Pressure Monitoring" in Medical Devices and Biosensors, 2008. ISSS-MDBS 2008. 5th International Summer School and Symposium on, 2008:28-32

[11] Bramwell JC, Hill AV, "The velocity of the pulse wave in man" in Proceedings of the Royal Society of London. Series B, Containing Papers of a Biological Character, 1922 April 1, Vol. 93, No. 652:298306

[12] Mikyoung Park, Hee Jung Kang, Young Huh et al.,"Cuffless and Noninvasive Measurement of Systolic Blood Pressure, Diastolic Blood Pressure, Mean Arterial Pressure and Pulse Pressure using Radial Artery Tonometry Pressure Sensor with Concept of Korean Traditional Medicine" in Engineering in Medicine and Biology Society, 2007. EMBS 2007. 29th Annual International Conference of the IEEE, 2007: $3597-3600$

[13] Mikyoung Park, Hee Jung Kang, Young Huh et al.,"Cuffless and Noninvasive Tonometry Mean Arterial Pressure Measurement by Physiological Characteristics and Applied Pressure" in Engineering in Medicine and Biology Society, 2007. EMBS 2006. 29th Annual International Conference of the IEEE, 2006: 6418 - 6421

[14] Healthstats Int., BPro [Online], 3.2.2011, http://www.healthstats.com/en/bpro.html

[15] Raamat R, Talts J, Jagomagi K. et al., "Effect of peripheral vasoconstriction on the beat-to-beat measurement of finger blood pressure" in Engineering in Medicine and Biology Society, 2000.
Proceedings of the 22nd Annual International Conference of the IEEE, 2000, vol.3:2223 - 2226

[16] O'Brien E, Waeber B, Parati G et al., „Blood pressure measuring devices: recommendations of the European Society of Hypertension." in BMJ, 2001 Mar 3;322(7285):531-6.

[17] Millan J, Park S-E, Kiefer S et al., „TOPCARE - Implementation of a telematic homecare platform in cooperative health care provider networks" in Engineering in Medicine and Biology, 2002. 24th Annual Conference and the Annual Fall Meeting of the Biomedical Engineering Society EMBS/BMES Conference, 2002. Proceedings of the Second Joint, 2002, vol.3:1869-1870

[18] Boqiang Liu, Yanyan Zhang, Zhongguo Liu, "Wearable monitoring system with multiple physiological parameters" in Medical Devices and Biosensors, 2008. ISSSMDBS 2008. 5th International Summer School and Symposium on, 2008 June 1-3:268-271

[19] Thomas G. Pickering, MD, DPhil; John E. Hall et al., "Recommendations for Blood Pressure Measurement in Humans and Experimental Animals. Part 1: Blood Pressure Measurement in Humans: A Statement for Professionals From the Subcommittee of Professional and Public Education of the American Heart Association Council on High Blood Pressure Research“" in Circulation, 2005 Feb 8;111(5):697716

[20] D'Angelo L.T., Czabke A., Somlai I., Niazmand K., Lüth T.C. (2010) : ART - A new Concept for an Activity Recorder and Transceiver, in 32nd Annual International Conference of the IEEE Engineering in Medicine and Biology Society, Medline, U.S. National Library of Medicine, pp. 2132-2135.

[21] Lüth, T.C., D'Angelo L.T., Czabke A. (2010) : TUM-AgeTech - A New Framework for Pervasive Medical Devices, in Pervasive and Smart Technologies for Healthcare: Ubiquitous Methodologies and Tools, editors Antonio Coronato and Giuseppe De Pietro, Hershey, USA, Medical Information Science Reference, pp. 295-321.

[22] D'Angelo L.T., Parlow J., Spiessl W., Hoch S., Lüth T.C. (2010) : A System for Unobtrusive In Car Vital Parameter Acquisition and Processing, in Pervasive Computing Technologies for Healthcare, 2010. PervasiveHealth 2010. Fourth International Conference on, pp. 1-7 